\title{
An Algorithm for Constructing Polynomial Systems Whose Solution Space Characterizes Quantum Circuits
}

\author{
Vladimir P. Gerdt and Vasily M. Severyanov \\ Laboratory of Information Technologies \\ Joint Institute for Nuclear Research \\ 141980 Dubna, Russia
}

\begin{abstract}
An algorithm and its first implementation in $\mathrm{C} \#$ are presented for assembling arbitrary quantum circuits on the base of Hadamard and Toffoli gates and for constructing multivariate polynomial systems over the finite field $Z_{2}$ arising when applying the Feynman's sum-over-paths approach to quantum circuits. The matrix elements determined by a circuit can be computed by counting the number of common roots in $Z_{2}$ for the polynomial system associated with the circuit. To determine the number of solutions in $Z_{2}$ for the output polynomial system, one can use the Gröbner bases method and the relevant algorithms for computing Gröbner bases.
\end{abstract}

Keywords: Quantum computation, Quantum circuit, Hadamard gate, Toffoli gate, Sum-over-paths approach, Polynomial equations, Finite field, Gröbner basis, Involutive algorithm, C\#

\section{INTRODUCTION}

When applying the famous Feynman's sum-over-paths approach to a quantum circuit, as it was shown in, ${ }^{1}$ one can associate with an arbitrary quantum circuit a polynomial system over the finite field $\mathbb{Z}_{2}$. For $N$ input qubits the system contains $N+1$ polynomials: each of the input qubits adds a certain polynomial to the system, and one more polynomial arises from the phase of a classical path through the circuit. Thereby, elements of the unitary matrix determined by the quantum circuit under consideration can be computed by counting the number of common roots in $Z_{2}$ for the polynomial system associated with the circuit. Given a quantum circuit, the polynomial set is uniquely constructed.

In this paper which is a substantially extended version of paper $^{2}$ we describe an algorithm for building an arbitrary quantum circuit made of Hadamard and Toffoli gates and for constructing the associated polynomial system. The algorithm implemented in the form of $\mathrm{C}^{6}$ program called QuPol (abbreviation of Quantum Polynomials). The program provides a user-friendly graphical interface for building quantum circuits and generating the associated polynomial systems.

To build an arbitrary quantum circuit, it is represented as a rectangular table with $N \times M$ cells, where $N$ the number of the input qubits, $M$ the number of the circuit cascades plus one - for the input $N$-qubit vector. Each cell contains an elementary gate taken from the elementary gate set. The last set of gates consists of wires (identities), arithmetic operations (multiplication and addition) and the Hadamard operation. A user of QuPol can construct a quantum circuit by selecting required elementary gates from the menu bar of the program and by placing them in the appropriate cells. As a result, the constructed table provides a visual representation of the circuit. A recursive evaluation procedure built-in $\mathrm{QuPol}$ operates on the table column-by-column and generates $N$ polynomials (one for each row of the table) as well as the phase polynomial in $n$ variables, where $n$ the number of Hadamard gates in the circuit.

To count the number of solutions in $Z_{2}$ for the output polynomial system, one can convert it into an appropriate Gröbner basis form which has the same number of solutions. Being invented 40 years ago, ${ }^{3}$ the Gröbner

Further author information: (Send correspondence to Vasily M. Severyanov)

Vladimir P.Gerdt: E-mail: gerdt@jinr.ru, Telephone: +7 (09621) 63437

Vasily M. Severyanov: E-mail: severyan@jinr.ru, Telephone: +7 (09621) 64800 
bases has become the most universal algorithmic method for analyzing and solving systems of polynomial equations. ${ }^{4}$ In particular, construction of the lexicographical Gröbner basis substantially alleviates the problem of the root finding for polynomial systems. To construct Gröbner bases one can use, for example, efficient involutive algorithms developed in. ${ }^{5}$ Our QuPol program together with a Gröbner basis software provides a tool to analyze quantum computation by applying methods of modern computational commutative algebra. We illustrate this tool by example from. ${ }^{1}$

The structure of the paper is as follows. In Section 2 we outline shortly the circuit model of quantum computation. Section 3 presents the famous Feynman's sum-over-paths method applied to quantum circuits. In Section 4 we consider a circuit decomposition in terms of the elementary gates and show how to build an arbitrary circuit composed from the Hadamard and Toffoli gates. These two gates form a universal gate basis. ${ }^{7,8}$ Some features of the algorithm implementation are briefly described. Section 5 demonstrates a simple example of handling the polynomials associated with a quantum circuit by constructing their Gröbner basis and computing the circuit matrix. We conclude in Section 6.

\section{QUANTUM CIRCUITS}

To compute a reversible Boolean vector-function $f: \mathbb{Z}_{2}^{n} \rightarrow \mathbb{Z}_{2}^{n}$, one applies an appropriate unitary transformation $U_{f}$ to an input state $|\mathbf{a}\rangle$ composed of of $n$ qubits $^{9}$ :

$$
|\mathbf{b}\rangle=U_{f}|\mathbf{a}\rangle, \quad|\mathbf{a}\rangle,|\mathbf{b}\rangle \in \mathbb{C}^{2^{n}} .
$$

The output state $|\mathbf{b}\rangle$ is generally not required result of the computation until somebody observes it. After that the output state becomes classical and can be used anywhere.

Some elementary unitary transformations are called quantum gates. A quantum gate acts only on a few qubits, on the remaining qubits it acts as the identity. One can build a quantum circuit by appropriately aligning quantum gates. In so doing, the unitary transformation of the circuit is the composition of its elementary unitary transformations:

$$
U_{f}=U_{m} U_{m-1} \cdots U_{2} U_{1} .
$$

A quantum gate basis is a set of universal quantum gates, i.e. any unitary transformation can be presented as a composition of the gates of the basis. As well as in the classical case, there can be different choice of the basis quantum gates. ${ }^{9}$ For our work it is convenient to choose the particular universal gate basis consisting of Hadamard and Toffoli gates. ${ }^{7,8}$

The Hadamard gate is a one-qubit gate. It turns the computational basis states into the equally weighted superpositions

$$
\begin{aligned}
& H:|0\rangle \mapsto \frac{1}{\sqrt{2}}(|0\rangle+|1\rangle), \\
& H:|1\rangle \mapsto \frac{1}{\sqrt{2}}(|0\rangle-|1\rangle)
\end{aligned}
$$

which differ in the phase factor at $|1\rangle$.

The Toffoli gate is a tree-qubit gate. The input bits $x$ and $y$ control the operation on bit $z$, and the Toffoli gate acts on the computational basis states as ${ }^{1}$

$$
(x, y, z) \mapsto(x, y, z \oplus x y) .
$$

An action of a quantum circuit can be described by a square unitary matrix whose entry $\left\langle\mathbf{b}\left|U_{f}\right| \mathbf{a}\right\rangle$ yields the probability amplitude for the transition from the initial quantum state $|\mathbf{a}\rangle$ to the final quantum state $|\mathbf{b}\rangle$. The matrix element is represented in accordance to the gate decomposition (1) of the circuit unitary transformation and can be calculated as sum over all the intermediate states $\mathbf{a}_{i}, \mathrm{i}=1,2, \ldots \mathrm{m}-1$ :

$$
\left\langle\mathbf{b}\left|U_{f}\right| \mathbf{a}\right\rangle=\sum_{\mathbf{a}_{i}}\left\langle\mathbf{b}\left|U_{m}\right| \mathbf{a}_{m-1}\right\rangle \cdots\left\langle\mathbf{a}_{1}\left|U_{1}\right| \mathbf{a}\right\rangle .
$$




\section{SUM-OVER-PATHS FOR QUANTUM CIRCUITS}

To apply the famous quantum-mechanical Feynman's sum-over-paths approach to calculation of the matrix elements for a quantum circuit, ${ }^{1}$ we replace each quantum gate in the circuit under consideration by its classical counterpart. The trick here is to determine the corresponding classical gate for the quantum Hadamard gate since its action at any input value 0 or 1 must generate 0 or 1 with the equal probability. To take this into account, the output of the classical Hadamard gate can be characterized by the path variable $x \in \mathbb{Z}_{2} \cdot{ }^{1}$ Its value determines one of the two possible paths of computation. Thereby, the classical Hadamard gate as

$$
a_{1} \mapsto x, \quad a_{i}, x \in \mathbb{Z}_{2},
$$

and the classical Toffoli gate acts as

$$
\left(a_{1}, a_{2}, a_{3}\right) \mapsto\left(a_{1}, a_{2}, a_{3} \oplus a_{1} a_{2}\right)
$$

where $\oplus$ denotes addition modulo 2 .

Fig. 1 shows an example of quantum circuit (taken from ${ }^{1}$ ) and its classical counterpart. The path variables $x_{i}$ comprise the (vector) path $\mathbf{x}=\left(x_{1}, x_{2}, x_{3}, x_{4}\right)^{T} \in \mathbb{Z}_{2}^{4}$.

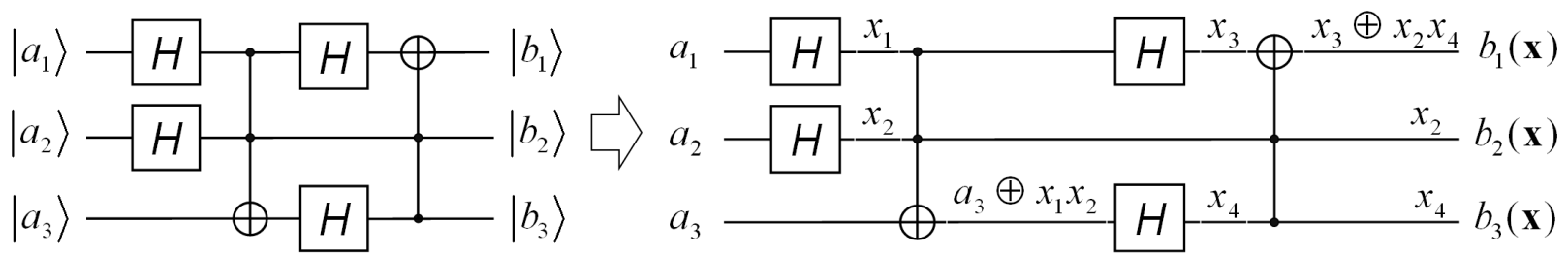

Figure 1. From quantum to classical circuit

A classical path is defined by a sequence of classical bit strings $\mathbf{a}, \mathbf{a}_{\mathbf{1}}, \mathbf{a}_{\mathbf{2}}, \ldots, \mathbf{a}_{\mathbf{m}}=\mathbf{b}$ obtained from application of the classical gates. Each set of values of the path variables $x_{i}$ gives a sequence of classical bit strings which is called an admissible classical path (Fig. 2). Each admissible classical path has a phase which is determined by

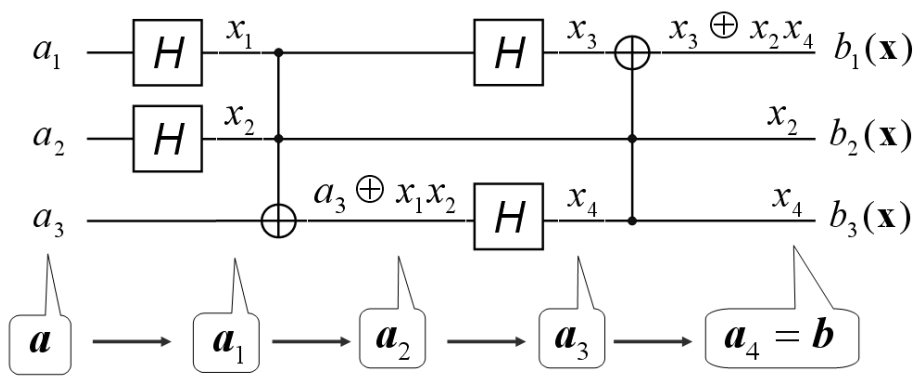

Figure 2. Admissible classical paths

the Hadamard gates applied. ${ }^{1}$ The phase is changed only when the input and output of the Hadamard gate are simultaneously equal to 1 , and this derives the formula

$$
\varphi(\mathbf{x})=\sum_{\text {Hadamard gates }} \text { input } \bullet \text { output } .
$$

with the sum evaluated in $\mathbb{Z}_{2}$. As to Toffoli gates, they do not change the phase. 
In our example the phase of the path $\mathbf{x}$ is

$$
\varphi(\mathbf{x})=a_{1} x_{1} \oplus a_{2} x_{2} \oplus x_{1} x_{3} \oplus x_{4}\left(a_{3} \oplus x_{1} x_{2}\right) .
$$

The matrix element of a quantum circuit is the sum over all the allowed paths from the classical state $\mathbf{a}$ to $\mathbf{b}$

$$
\left\langle\mathbf{b}\left|U_{f}\right| \mathbf{a}\right\rangle=\frac{1}{\sqrt{2^{h}}} \sum_{\mathbf{x}: \mathbf{b}(\mathbf{x}) \mathbf{b}}(-1)^{\varphi(\mathbf{x})}
$$

where $h$ is the number of Hadamard gates. The terms in the sum have the same absolute value but may vary in sign.

Let $N_{0}$ be the number of positive terms in the sum and $N_{1}$ be the number of negative terms:

$$
\begin{aligned}
& N_{0}=|\{\mathbf{x} \mid \mathbf{b}(\mathbf{x})=\mathbf{b} \wedge \varphi(\mathbf{x})=0\}|, \\
& N_{1}=|\{\mathbf{x} \mid \mathbf{b}(\mathbf{x})=\mathbf{b} \wedge \varphi(\mathbf{x})=1\}| .
\end{aligned}
$$

Hence, $N_{0}$ and $N_{1}$ count the number of solutions for the indicated systems of $n+1$ polynomials in $h$ variables over $\mathbb{Z}_{2}$. Then the matrix element may be written as the difference

$$
\left\langle\mathbf{b}\left|U_{f}\right| \mathbf{a}\right\rangle=\frac{1}{\sqrt{2^{h}}}\left(N_{0}-N_{1}\right) .
$$

\section{ALGORITHM AND PROGRAM}

For building arbitrary quantum circuits from Hadamard and Toffoli gates, we use the set of elementary gates

$$
E=\{I, \stackrel{+}{I}, \stackrel{\wedge}{I}, \stackrel{\vee}{I}, \stackrel{\wedge}{M}, \stackrel{\vee}{M}, \stackrel{\wedge}{A}, \stackrel{\vee}{A}, H\}
$$

shown on Fig. 3. Furthermore, we represent a circuit as a rectangular table (Fig. 4, left).

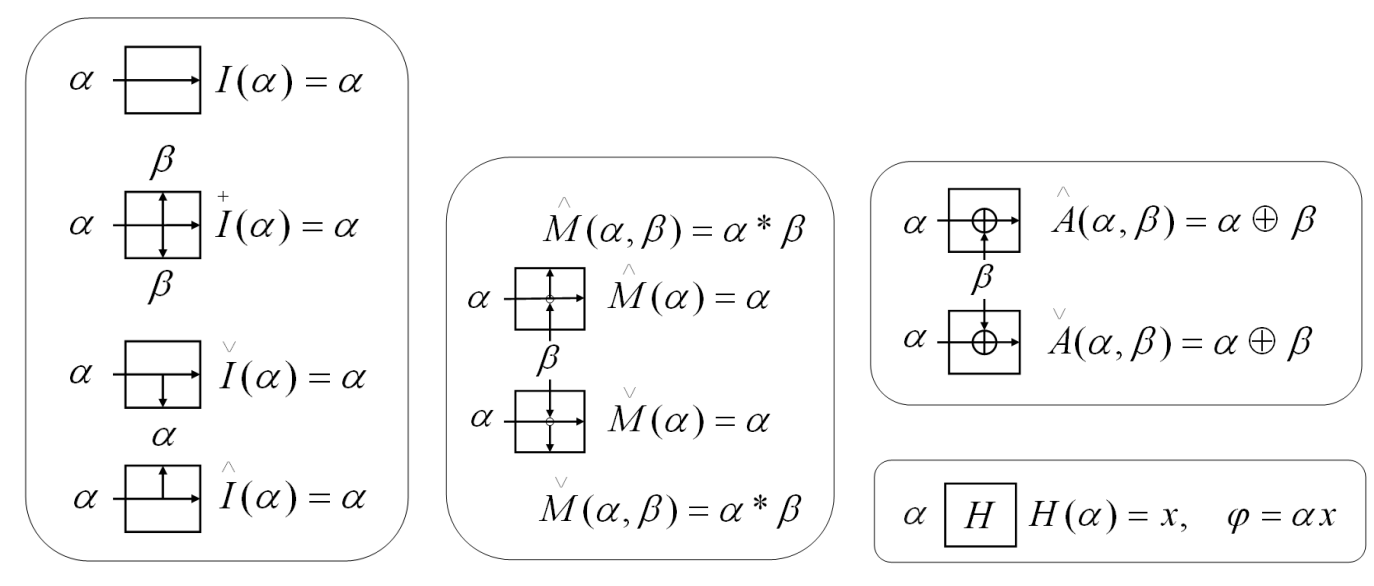

Figure 3. Elementary gates

Each cell in the table contains an elementary gate so that the output for each row is determined by the composition of the elementary gates in the row (taking into account recursive dependences). Hence, each elementary unitary transformation $U_{j}$ is represented by an $n$-tuple of elementary gates.

Fig. 3 shows action of the elementary gates from the set (5): the identities, the multiplications, the additions modulo 2, and the classical Hadamard gate. The identity $I$ just reproduces its input. The identity-cross 

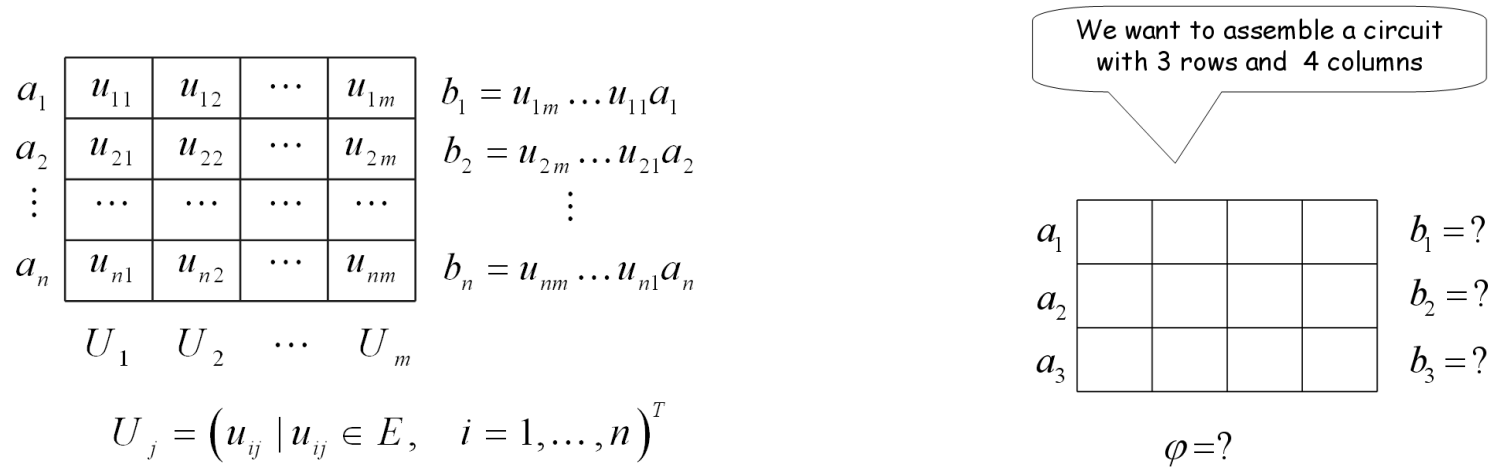

Figure 4. Decomposition into elementary gates (left) and building a sample circuit, step 0 (right)
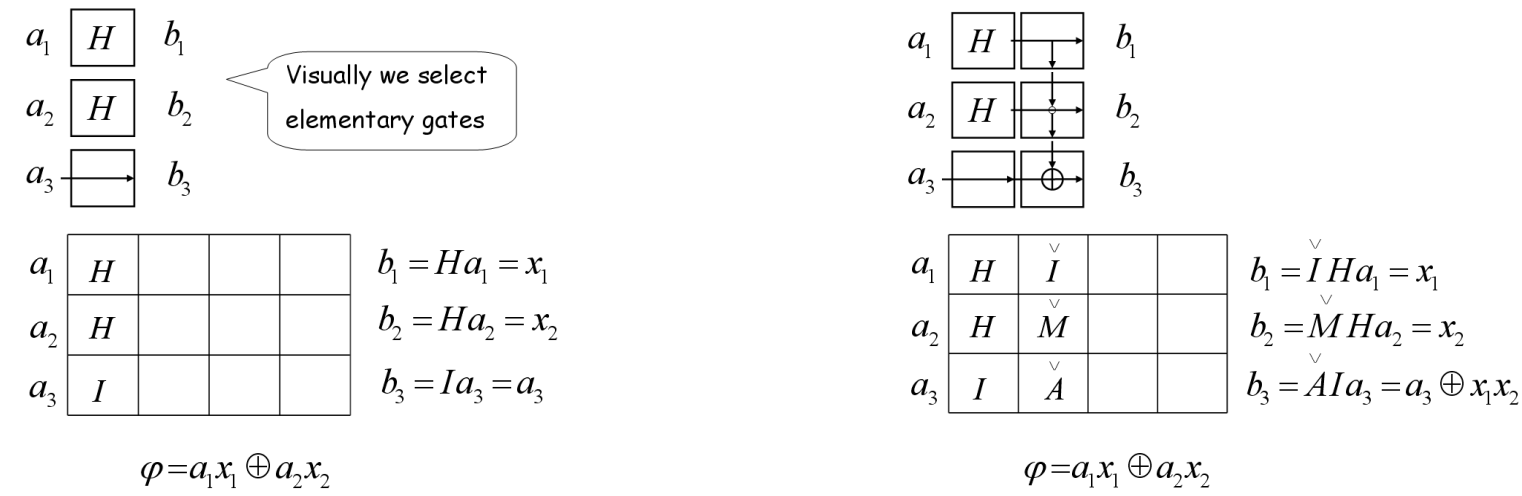

$\varphi=a_{1} x_{1} \oplus a_{2} x_{2}$

Figure 5. Building circuit, steps 1 (left) and 2 (right)

$\stackrel{+}{I}$ reproduces also its vertical input from the top elementary gate to the bottom one and vice-versa. Every identity-down $\stackrel{\vee}{I}$ and identity-up $\hat{I}$ have two outputs - horizontal and vertical. The multiplication-up $\hat{M}$ and multiplication-down $\stackrel{\vee}{M}$ perform multiplication of their horizontal and the corresponding vertical inputs. The addition-up $\hat{A}$ and addition-down $\stackrel{\vee}{ }$ are applied similarly. Each Hadamard gate outputs an independent path variable irrespective of its input and can give a nonzero contribution to the phase.

To build a circuit, we define, first, an empty table of the required size (Fig. 4, right). At this initialization step the output qubit values and phase are not determined. As the second step we place the required elementary gates in appropriate cells of the first column and construct the circuit polynomials (Fig. 5, left). Now the output is obtained by applying the inserted elementary gates to the input. The phase is also calculated. Then we proceed the same way with the second column (Fig. 5, right), then with the third and forth columnns (Fig. 6).

A circuit is represented in our program QuPol as two $2 d$-arrays: one for the elementary gates and another for the related polynomials. The phase polynomial is separately represented. The following piece of code demonstrates construction of the circuit polynomials

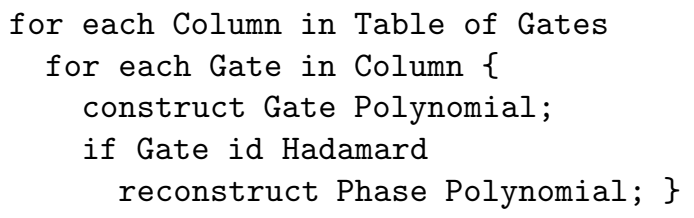




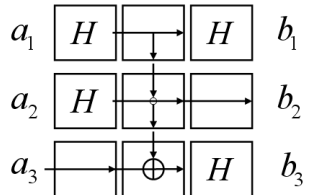

\begin{tabular}{|c|c|c|c|c|}
\hline$a_{1}$ & $H$ & $I$ & $H$ & $b_{1}=H I H a_{1}=x_{3}$ \\
\hline$a_{2}$ & $H$ & $M$ & $I$ & $b_{2}=I M H a_{2}=x_{2}$ \\
\hline$a_{3}$ & $I$ & $A$ & $H$ & $b_{3}=H A I a_{3}=x_{4}$ \\
\hline
\end{tabular}

$\varphi=a_{1} x_{1} \oplus a_{2} x_{2} \oplus x_{1} x_{3} \oplus x_{4}\left(a_{3} \oplus x_{1} x_{2}\right)$

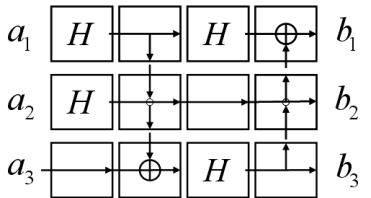

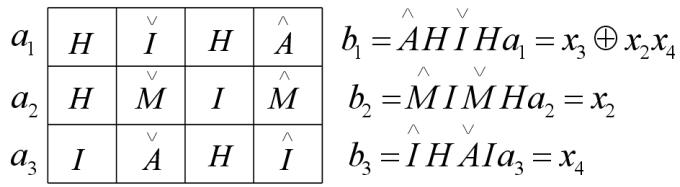

$\varphi=a_{1} x_{1} \oplus a_{2} x_{2} \oplus x_{1} x_{3} \oplus x_{4}\left(a_{3} \oplus x_{1} x_{2}\right)$

Figure 6. Building circuit, steps 3 (left) and 4 (right)

It should be noticed that Figs. 5 and 6 show a shortened version of the elementary gates composition. Actually, the final polynomials look like:

$$
\begin{aligned}
b_{1} & =\hat{A}\left(H \stackrel{\vee}{H} H a_{1}, \stackrel{\wedge}{M}\left(I \stackrel{\vee}{M}\left(H a_{2}, \stackrel{\vee}{I} H a_{1}\right), \stackrel{\wedge}{I} H \stackrel{\vee}{A}\left(I a_{3}, \stackrel{\vee}{M}\left(H a_{2}, \stackrel{\vee}{I} H a_{1}\right)\right)\right)\right)=x_{3} \oplus x_{2} x_{4}, \\
b_{2} & =\hat{M}\left(I \stackrel{\vee}{M}\left(H a_{2}, \stackrel{\vee}{I} H a_{1}\right), \stackrel{\wedge}{I} H \stackrel{\vee}{A}\left(I a_{3}, \stackrel{\vee}{M}\left(H a_{2}, \stackrel{\vee}{I} H a_{1}\right)\right)\right)=x_{2}, \\
b_{3} & =\hat{\sim} H \stackrel{\vee}{A}\left(I a_{3}, \stackrel{\vee}{M}\left(H a_{2}, \stackrel{\vee}{I} H a_{1}\right)\right)=x_{4} .
\end{aligned}
$$

The method for constructing a gate polynomial is essentially recursive because of the need to go up or down for some gates.

Any circuit is saved in the program as two files. One file is binary and contains the circuit itself. Another file has a text format. It contains the circuit polynomials in a symbolic form. The program allows one to save polynomials in several formats suitable as inputs for computer algebra systems such as Maple or Mathematica for the further processing. It is also possible to load the saved circuit back into the computer memory for its modification.

The part of our code for the name space Polynomial_Modulo_2 written in C\# can be also used indepen-

\section{Class Polynomial: IEnumerable}

- void AddTo (Polynomial polynomial)
- void MultiplyBy (Polynomial polynomial)
- override string ToString ()
- bool FromString (string str)
- float AsGraphics ( . . . )
- void Collect () - collect terms
- int Count () - get number of monomials

Figure 7. Class Polynomial 
dently on our program. This name space contains a set of classes for handling polynomials over the field $\mathbb{Z}_{2}$. Class Polynomial (Fig. 7) is a list of monomials, class Monomial (Fig. 8) is a list of letters, class Letter (Fig. 9)

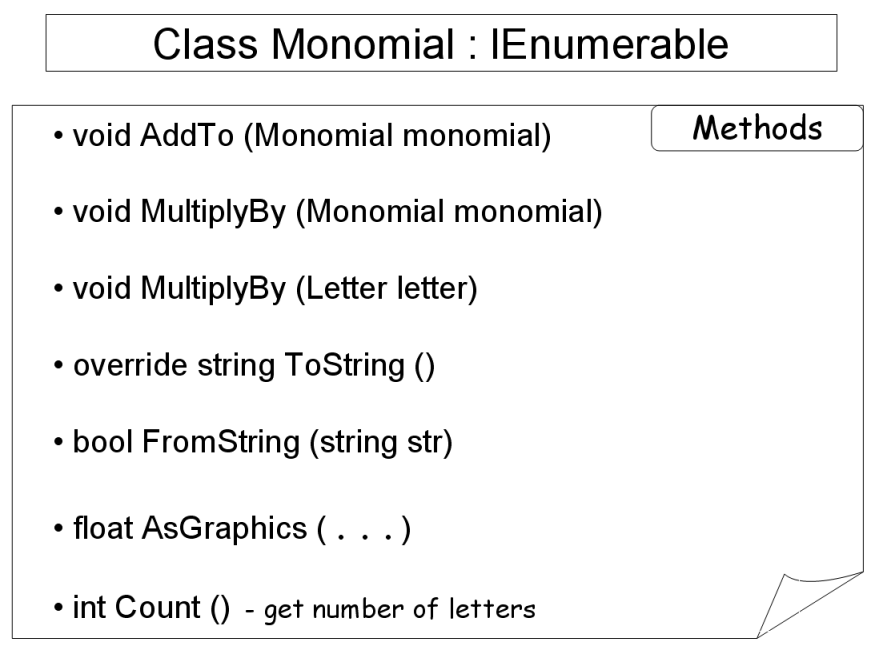

Figure 8. Class Monomial

is an indexed letter provided with a positive integer superscript (power degree).

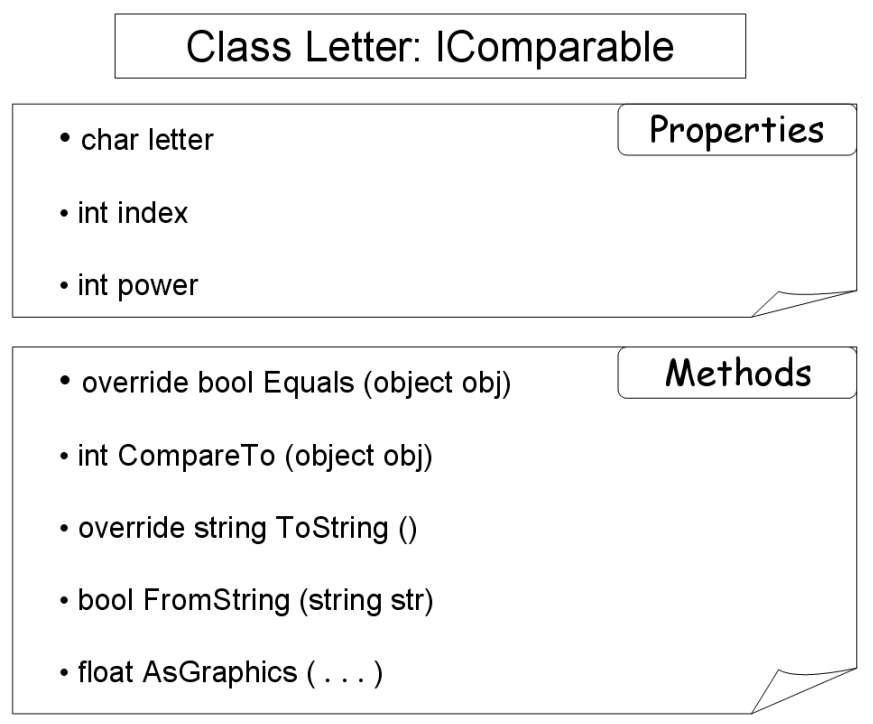

Figure 9. Class Letter

\section{CALCULATING CIRCUIT MATRIX ELEMENTS}

A system generated by the program is a finite set $F \subset R$ of polynomials in the ring

$$
R=\mathbb{Z}_{2}\left[a_{i}, b_{j}\right]\left[x_{1}, \ldots, x_{h}\right], \quad a_{i}, b_{j} \in \mathbb{Z}_{2}, \quad i, j=1, \ldots, n
$$


with $h$ polynomial variables and $2 n$ binary coefficients. Let $N_{0}$ and $N_{1}$ denote, as in (2-3), the number of roots in $\mathbb{Z}_{2}$ of the polynomial sets $F_{0}$ and $F_{1}$, respectively, where

$$
F_{0}=\left\{f, \ldots, f_{k}, \varphi\right\}, \quad F_{1}=\left\{f, \ldots, f_{k}, \varphi \oplus 1\right\} .
$$

Then the circuit matrix is given as

$$
\langle\mathbf{b}|U| \mathbf{a}\rangle=\frac{1}{\sqrt{2^{h}}}\left(N_{0}-N_{1}\right)
$$

where $h$ is the number of Hadamard gates in the circuit.

To compute $N_{0}$ and $N_{1}$ one can convert $F_{0}$ and $F_{1}$ into an appropriate triangular form ${ }^{10}$ providing elimination of the path variables $x_{1}, \ldots, x_{h}$. One of such triangular forms is the pure lexicographical Gröbner basis that can be computed, for instance, by means of the Buchberger algorithm, ${ }^{3,4}$ by the Faugère algirthms ${ }^{11,12}$ or by our involutive algorithm. ${ }^{5}$

For the circuit shown on Fig. 1 we obtain the following polynomial set:

$$
\begin{aligned}
& f_{1}=x_{2} x_{4} \oplus x_{3} \oplus b_{1}, \\
& f_{2}=x_{2} \oplus b_{2}, \\
& f_{3}=x_{4} \oplus b_{3}, \\
& \varphi=x_{1} x_{2} \oplus x_{1} x_{3} \oplus a_{1} x_{1} \oplus a_{2} x_{2} \oplus a_{3} x_{4} .
\end{aligned}
$$

The lexicographical Gröbner bases for polynomial systems $F_{0}$ and $F_{1}$ and for the ordering $x_{1} \succ x_{2} \succ x_{3} \succ x_{4}$ on the variables are given by

$$
G_{0}:\left\{\begin{array}{l}
g_{1}=\left(a_{1} \oplus b_{1}\right) x_{1} \oplus a_{2} b_{2} \oplus a_{3} b_{3}, \\
g_{2}=x_{2} \oplus b_{2}, \\
g_{3}=x_{3} \oplus b_{1} \oplus b_{2} b_{3}, \\
g_{3}=x_{4} \oplus b_{3},
\end{array} \quad G_{1}:\left\{\begin{array}{l}
g_{1}=\left(a_{1} \oplus b_{1}\right) x_{1} \oplus a_{2} b_{2} \oplus a_{3} b_{3} \oplus 1 \\
g_{2}=x_{2} \oplus b_{2}, \\
g_{3}=x_{3} \oplus b_{1} \oplus b_{2} b_{3}, \\
g_{3}=x_{4} \oplus b_{3} .
\end{array}\right.\right.
$$

These lexicographical Gröbner bases immediately yield the following conditions on the parameters:

$$
\begin{array}{ll}
G_{0}: & a_{1} \oplus b_{1}=a_{2} b_{2} \oplus a_{3} b_{3}=0, \\
G_{1}: & a_{1} \oplus b_{1}=0 \wedge a_{2} b_{2} \oplus a_{3} b_{3}=1 .
\end{array}
$$

It is immediately follows that if conditions (10) are satisfied then the polynomial system $G_{0}$ (resp. $F_{0}$ ) has two common roots in $\mathbb{Z}_{2}$ and $G_{1}$ (resp. $F_{1}$ ) has no common roots, and, vise-versa, if conditions (10) are satisfied then $G_{0}$ has no roots and $G_{1}$ has two roots. In all other cases there is one root of $G_{0}$ and one root of $G_{1}$.

In that way, the $8 \times 8$ matrix for the circuit of Fig.1 is easily determined by formulae (4) with $N_{0}$ and $N_{1}$ defined from systems (9). Table 1 explicitly shows entries of this matrix when its rows and columns are indexed by the values of the input bits $a_{1}, a_{2}, a_{3}$ and output bits $b_{1}, b_{2}, b_{3}$, respectively.

\section{CONCLUSION}

We presented an algorithm for building an arbitrary quantum circuit composed of Hadamard and Toffoli gates and for constructing the corresponding polynomial equation systems over $\mathbb{Z}_{2}$. The number of common roots $\mathbb{Z}_{2}$ of polynomials in the system uniquely determines the circuit matrix. The algorithm has been implemented in as a C\#-program QuPol.

To count the number of polynomial roots one can use the universal algorithmic Gröbner basis approach. In the framework of this approach the polynomial system under consideration is converted into a triangular form that is convenient for computing the number of roots. 


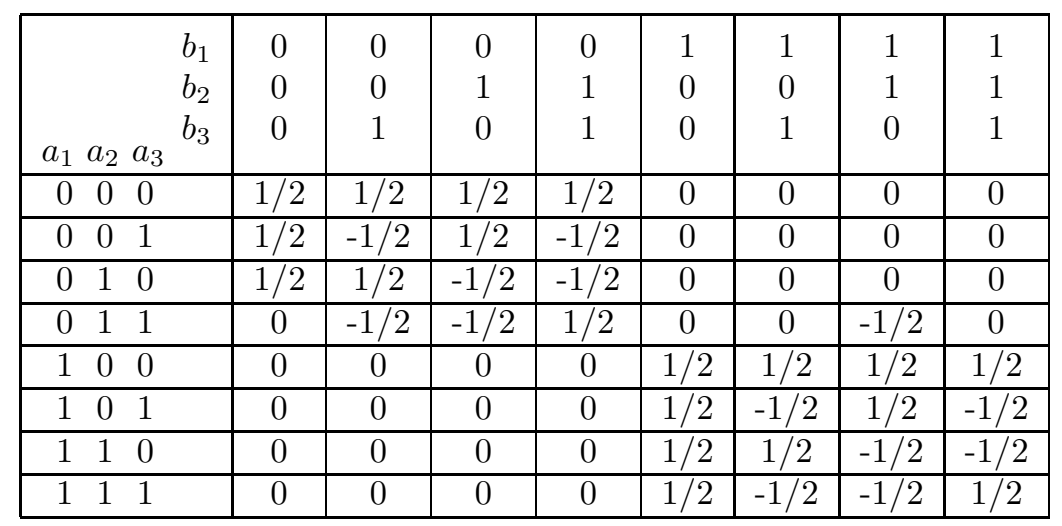

Table 1. The circuit matrix for polynomial system (8)

General theoretical complexity bound for computing Gröbner bases (see, for instance, ${ }^{13}$ ) is double exponential in the number of polynomial variables $x_{i}$ (the number of path variables, i.e. Hadamard gates, in our case). However, the due to the fact that all the path variables $x_{i}$, the input and output parameters $a_{j}, b_{k}$ as well as the numerical coefficients of the polynomials are elements in the finite field $\mathbb{Z}_{2}$ the Gröbner basis computation is sharply simplified. Thus, based on the algorithm in, ${ }^{12}$ the analysis of costs for computing Gröbner bases for polynomials over $Z_{2}$, arising in cryptography, revealed ${ }^{14}$ only a single exponential complexity in the number of equations.

Therefore, the above presented algorithm together with an appropriate software for computing a Gröbner basis and for counting its number of common roots in $\mathbb{Z}_{2}$ provides a tool for simulating quantum logical circuits and for estimation of their computational power. For this purpose we are planning to create a special module in the open source software GINV. ${ }^{16}$ At present, GINV contains implementation in $\mathrm{C}++$ of our involutive algorithm $^{5}$ for computing Gröbner bases over the field of rational numbers and over the field $\mathbb{Z}_{p}$ where $p$ is a prime number. By this reason, the current version of GINV can be used for computing Gröbner bases for polynomial systems (7) only if one considers $2 n$ parameters $a_{i}, b_{j}$ in (6) as variables. In this case, to compute a triangular Gröbner basis form of the circuit polynomial, one should use an elimination term order for those parameters such that any term containing path variables $x_{k}$ is higher than that any term containing parameters only.

Such computational scheme, however, increases the number of polynomial (path) variables by $2 n$ what may lead to exponential slowing down, as was pointed to above. That is why we expect that an adaptation of the built-in algorithms and data structures to the polynomial ring (6) with parametric coefficients will substantially increase computational efficiency of GINV in its application to analysis and simulation of quantum circuits.

Recently another algorithmic approach was suggested ${ }^{15}$ to work with polynomial systems over finite fields. It seems interesting to investigate computational efficiency of the approach in ${ }^{15}$ in comparison with that based on Gröbner bases.

\section{ACKNOWLEDGMENTS}

The research presented in this paper was partially supported by the grant 04-01-00784 from the Russian Foundation for Basic Research.

\section{REFERENCES}

1. C.M. Dawson et al. Quantum computing and polynomial equations over the finite field $Z_{2}$. arXiv:quant$\mathrm{ph} / 0408129$.

2. V.P.Gerdt and V.M.Severyanov. A Software Package to Construct Polynomial Sets over $Z_{2}$ for Determining the Output of Quantum Computations. arXiv:quant-ph/0509064. 
3. B.Buchberger. An Algorithm for Finding a Basis for the Residue Class Ring of a Zero-Dimensional Polynomial Ideal. PhD Thesis, University of Innsbruck, 1965 (in German).

4. B.Buchberger and F.Winkler (eds.) Gröbner Bases and Applications. Cambridge University Press, 1998.

5. V.P.Gerdt. Involutive Algorithms for Computing Gröbner Bases. In: "Computational commutative and noncommutative algebraic geometry", IOS Press, Amsterdam, 2005, pp. 199-225. arXiv:math.AC/0501111.

6. Microsoft Visual C\# .net Standard, Version 2003.

7. Y.Shi. Both Toffoli and Control-Not need little help to do universal quantum computation, Quantum Information and Computation, 3(1):84-92, 2003. arXiv:quant-ph/0205115.

8. D.Aharonov. A Simple Proof that Toffoli and Hadamard Gates are Quantum Universal. arXiv:quant$\mathrm{ph} / 0301040$.

9. M.A.Nielsen and I.L.Chuang. Quantum Computation and Quantum Information. Cambridge University Press, Cambridge, 2000.

10. D.Wang. Elimination Methods. Springer-Verlag, Berlin, 1999.

11. J.-C. Faugère. A new efficient algorithm for computing Gro"bner bases (F4), Journal of Pure and Applied Algebra, 139(1-3): 61-88, 1999.

12. J.-C.Faugère. A new efficient algorithm for computing Gröbner bases without reduction to zero ( $\left.F_{5}\right)$. In: "Proceedings of Issac 2002", ACM Press, New York, 2002, pp. 75-83.

13. J. von zur Garthen and J.Gerhard. Modern Computer Algebra. 2nd edition, Cambridge University Press, 2003.

14. Project-Team Spaces. Solving Problems through Algebraic Computation and Efficient Software. Activity Report, INRIA, 2003, p.13. http://www.inria.fr/rapportsactivite/RA2003/

15. V.V.Kornyak. On Compatibility of Discrete Relations. Lecture Notes in Computer Science. Springer-Verlag, Berlin, 2005, pp.272-284. arXiv:org/math-ph/0504048.

16. http://invo.jinr.ru 